\title{
The Role of Conformational Flexibility in Baeyer-Villiger Monooxygenase Catalysis and Structure
}

Brahm J. Yachnin ${ }^{\mathrm{a}, \mathrm{e}}$, Peter C. K. Lau, ${ }^{\mathrm{c}, \mathrm{f},}$, Albert M. Berghuis ${ }^{\mathrm{a}, \mathrm{b}, \mathrm{e}, *}$

Departments of a Biochemistry and ${ }^{b}$ Microbiology \& Immunology, McGill University, 3649

Promenade Sir William Osler, Bellini Pavilion, Room 466, Montreal, Quebec, Canada, H3G 0B1 


\section{ABSTRACT}

Background: The Baeyer-Villiger monooxygenases (BMVOs) are a group of microbial enzymes that have garnered interest as industrial biocatalysts. While great strides have been made in recent years to understand the mechanism of these enzymes from a structural perspective, our understanding remains incomplete. In particular, the role of a twenty residue loop (residues 487504), which we refer to as the "Control Loop," that is observed in either an ordered or disordered state in various crystal structures remains unclear.

Methods: Using SAXS, we have made the first observations of the Loop in solution with two BVMOs, cyclohexanone monooxygenase (CHMO) and cyclopentadecanone monooxygenase. We also made a series of mutants of CHMO and analysed them using SAXS, ITC, and an uncoupling assay.

Results: These experiments show that Control Loop ordering results in an overall more compact enzyme without altering global protein foldedness. We have also demonstrated that the Loop plays a critical and complex role on enzyme structure and catalysis. The Control Loop appears to have a direct impact on the organization of the overall structure of the protein, as well as in influencing the active site environment.

Conclusions: The data imply that the Loop can be divided into two regions, referred to as "subloops," that coordinate overall domain movements to changes in the active site.

General Significance: A better understanding of the mechanistic role of the Control Loop may ultimately be helpful in designing mutants with altered specificity and improved catalytic efficiency.

Keywords: Baeyer-Villiger monooxygenase; small-angle X-ray scattering (SAXS); isothermal titration calorimetry (ITC); protein flexibility; enzyme mechanism; enzyme catalysis

Abbreviations: AcCHMO, cyclohexanone monooxygenase from Acinetobacter sp. NCIMB 9871; BVMO, Baeyer-Villiger monooxygenase; BVO, Baeyer-Villiger oxidation; CHMO, cyclohexanone monooxygenase; CPDMO, cyclopentadecanone monooxygenase; $\mathrm{D}_{\max }$, maximum interatomic distance; ITC, isothermal titration calorimetry; $\mathrm{K}_{\mathrm{d}}$, dissociation constant; OTEMO, 2-oxo- $\Delta^{3}-4,5,5$-trimethylcyclopentenylacetyl-coenzyme A monooxygenase; PAMO, phenylacetone monooxygenase; $\mathrm{R}_{\mathrm{g}}$, radius of gyration; $R \mathrm{mCHMO}$, cyclohexanone monooxygenase from Rhodococcus sp. HI-31; SAXS, small angle X-ray scattering; TCEP, Tris(2-carboxy)phosphine; $\mathrm{V}_{\mathrm{c}}$, volume-of-correlation. 


\section{INTRODUCTION}

The Baeyer-Villiger monooxygenases (BVMOs) are a group of microbial flavoproteins that are able to catalyze the synthetically useful Baeyer-Villiger oxidation (BVO) reaction, which involves the conversion of ketones into esters or cyclic ketones to lactones $[1,2]$. Since their discovery in the 1970s [3, 4], these enzymes have been increasingly looked upon as preferred catalysts for accessing chiral lactones for synthetic purposes [5-7]. Chemo-, regio-, and enantioselectivities are hallmarks of BVMOs, as is their use of molecular oxygen as oxidant, rather than hazardous organic peracids in typical BVO catalysis. A prototypical BVMO is the NADPHdependent cyclohexanone monooxygenase (CHMO) from Acinetobacter sp. NCIMB 9871 (AcCHMO), the mechanism of which was extensively studied as early as the 1980s [8]. From this study, as well as a later mechanistic study of AcCHMO [9], the BVMOs were found to have a complex, highly ordered enzyme mechanism. In the conversion of cyclohexanone to $\varepsilon^{-}$ caprolactone, the first step in this reaction cycle involves the binding of NADPH, which reduces the tightly bound FAD molecule in the core of the protein. Molecular oxygen then binds to the enzyme and reacts with the reduced flavin to form the peroxyanion intermediate. The ketone substrate binds last, reacts with the peroxyanion intermediate, and forms the tetrahedral Criegee intermediate, which is analogous to the Criegee intermediate that is formed in the chemical BVO reaction [10]. The Criegee intermediate then rearranges to form the lactone product. Following dehydration of the flavin, the lactone is released back to the solvent, and finally $\mathrm{NADP}^{+}$is released. This ordered mechanism was also observed in another BVMO, the thermostable phenylacetone monooxygenase (PAMO) [11], suggesting that it is likely to be a general mechanism for all BVMOs.

The first crystal structure of a BVMO was reported a decade ago, with the publication of the FAD-bound structure of PAMO [12]. Even at this early stage, the importance of protein flexibility and conformational changes was apparent, as a strictly conserved arginine, R337, was found to be in multiple conformations that were believed to be associated with domain rotations of the enzyme. In addition, a large loop was observed to be protruding from the protein surface and contacting a symmetry-related protein molecule in the crystal structure. This interaction was believed to be a crystallization artifact, implying that the loop was unstructured in solution. Another noteworthy observation from this first structure was that the strictly conserved BVMO 
signature sequence, consisting of the sequence FxGxxxHxxxW(P/D) [13, 14], was found to be distant from the active site. As this sequence's importance has been highlighted in identifying many new BVMOs, its distance from the active site suggested that it must be playing an important structural role.

This structure was followed by two $\mathrm{NADP}^{+}$-bound crystal structures of CHMO from Rhodococcus sp. HI-31 (RmCHMO) [15]. These structures introduced additional conformations in the conserved arginine residue (R329 in RmCHMO). It also allowed the enzyme to be divided into three domains, known as the NADPH-binding domain, the FAD-binding domain, and the helical domain, owing to the pronounced rotation of the NADPH-binding domain when comparing these structures. In addition, the large loop (residues 487-504 in RmCHMO) was found to be disordered in the first structure, known as $\mathrm{CHMO}_{\text {Open, }}$, and folded in and contacting

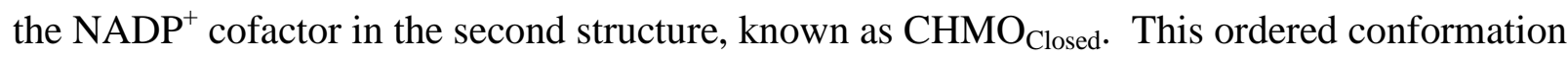
of the loop was unlikely to be a crystallization artifact, as mutation of W492, which interacts with the $\mathrm{NADP}^{+}$molecule, resulted in an enzyme with drastically reduced activity. Surprisingly, the W492A mutant was also found to increase the stereospecificity of the enzyme, albeit at the cost of enzyme efficiency, again highlighting the importance of this loop [16]. Due to the apparent impact of this loop on structure, activity, and stereospecificity based on both these previous studies as well as the present study, we will refer to this loop spanning residues 487-504 as the "Control Loop" (or "Loop") hereafter.

More recently, crystal structures of PAMO with $\mathrm{NADP}^{+}$bound have corroborated the Control Loop order-disorder transition observed in $\mathrm{RmCHMO}$, as well as the observed domain rotations [17]. Crystal structures of 2-oxo- $\Delta^{3}-4,5,5$-trimethylcyclopentenylacetyl-coenzyme A monooxygenase (OTEMO) from a camphor degradation pathway have demonstrated alternate Control Loop configurations that are required to accommodate the very large, coenzyme A linked substrates of this enzyme [18]. Finally, substrate-bound [19] and product-bound [16] crystal structures of $\mathrm{RmCHMO}$, known as $\mathrm{CHMO}_{\text {Rotated }}, \mathrm{CHMO}_{\text {Loose }}$, and $\mathrm{CHMO}_{\text {Tight }}$, highlighted the multiple conformations of $\mathrm{NADP}^{+}$, the domain rotations, and the ability of the Control Loop to adopt either the disordered conformation (seen in $\mathrm{CHMO}_{\text {Rotated }}$ and $\mathrm{CHMO}_{\text {Loose }}$ ) or the ordered, folded-in conformation (seen in $\mathrm{CHMO}_{\text {Tight }}$ ) with the same ligands bound. The catalytic mechanism proposed based on these crystal structures suggests that the enzyme requires that the 
Loop undergoes multiple order-disorder transitions per catalytic cycle. The structural framework for $\mathrm{BVMO}$ stereospecificity based on the $\mathrm{CHMO}_{\text {Tight }}$ structure has since been used to successfully alter the stereospecificity of two CHMO enzymes [20].

That the Control Loop has a role in catalysis appears to have been confirmed by the changes observed in the W492A mutant $[15,16]$. The nature of this role, however, is not clear. It is possible that it simply acts to prevent dissociation of $\mathrm{NADP}^{+}$and/or the substrate during the catalytic cycle; however, the link between the degree of rotation of the NADPH-binding domain and the Loop order/disorder state implies a more complex role. Examination of the Loop contacts (Fig. 1) shows that in addition to the interaction of W492 with $\mathrm{NADP}^{+}$, the backbone carbonyls of G489 and D490 interact with K328 (Fig. 1c). K328 is a notable residue, as it forms a small loop with the conserved R329. As R329 changes conformation in response to the active site configuration, this links the Control Loop to the active site at a second position. As this part

a
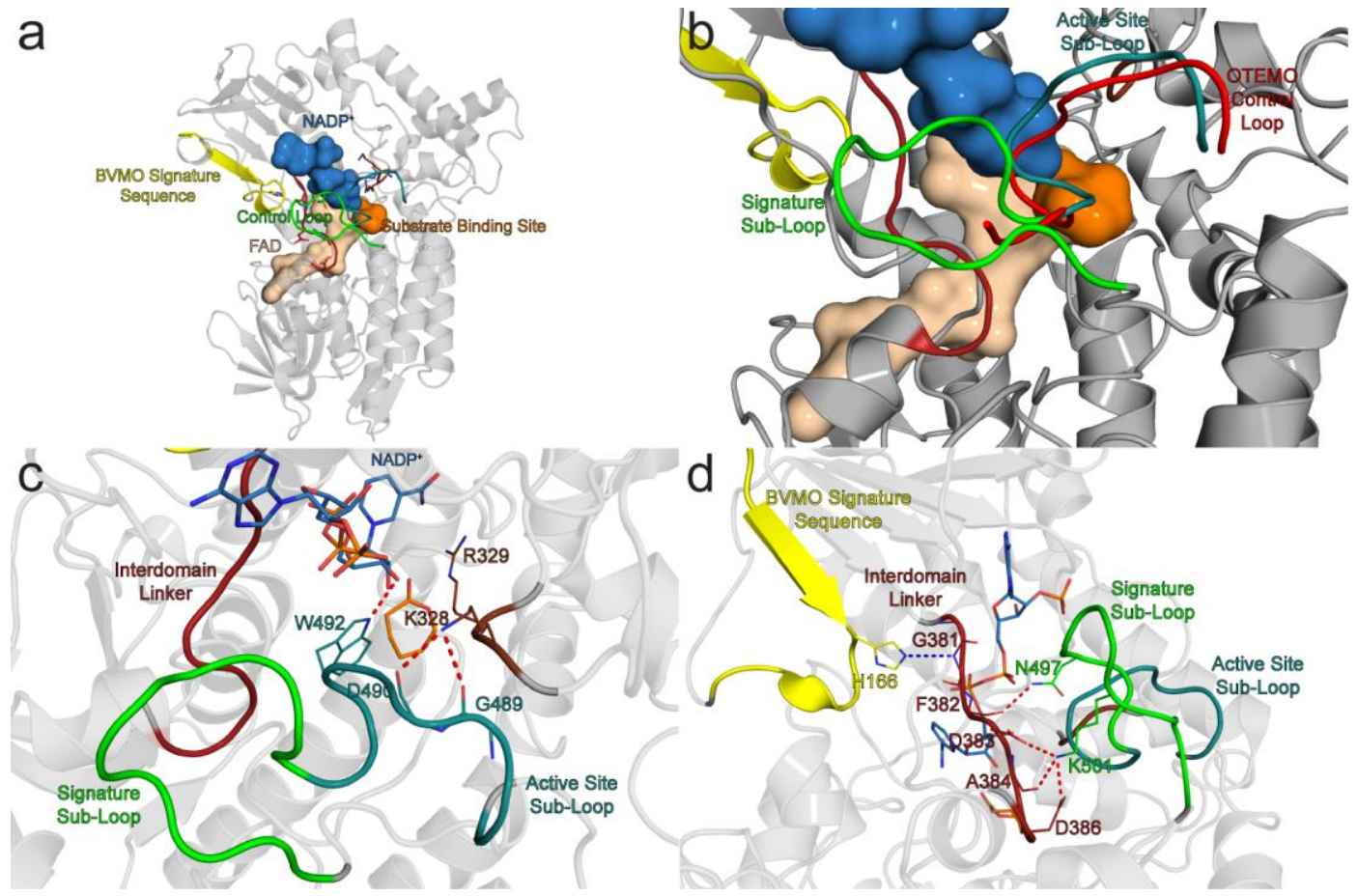

Fig. 1. The structure of the Control Loop. (a) The overall structure of CHMO is shown, with the Control Loop (residues 487-504) and BVMO signature sequence (residues 160-171) highlighted. (b) Comparison of the Control Loop in CHMO and OTEMO. The signature sub-loop is disordered in the OTEMO crystal structure, and not shown. (c) The key interactions between the active site and active site sub-loop (residues 487-494) are shown. (d) The key interactions between the signature sub-loop (residues 495-504), the interdomain linker (residues 378-386), and the BVMO signature sequence are shown. In all panels, the BVMO signature sequence is shown in yellow, the signature sub-loop is shown in green, the active site sub-loop is shown in teal, the interdomain linker is shown in dark red, and the K328/R329 loop is shown in brown. NADP ${ }^{+}$is shown in blue, FAD is shown in wheat, and the substrate binding site is shown in orange. PDB ID 4RG3 is used in all panels. The OTEMO structure shown in panel B is PDB ID 3UP4, chain A. 
of the Loop, roughly spanning residues $487-494$, is in close proximity to the active site, we will refer to it as the "active site sub-loop."

The Control Loop also may be playing a further structural role (Fig. 1d). The Loop residues N497 and K501 interact through a number of residues to an interdomain linker spanning residues 378-386. This linker includes G381, which interacts with H166 of the BVMO signature sequence. We previously suggested that this interaction may be an "atomic switch" that is turned on only in the presence of $\mathrm{NADP}^{+}[15]$. This may indicate that the Control Loop is linked to the BVMO signature sequence. As such, we will refer to this part of the Loop, roughly spanning residues 495-504, as the "signature sub-loop." Intriguingly, OTEMO, which requires a different active site environment to accommodate its coenzyme A-linked substrates, has the signature subloop disordered even when residues 489, 490, and 492 are ordered and engaged in the interactions observed in the $\mathrm{CHMO}_{\text {Closed }}$ crystal structure (Fig. 1b) [18].

In order to complement the series of crystal structures of BVMOs that have been studied, here we report a series of small-angle X-ray scattering (SAXS), isothermal titration calorimetry (ITC), and uncoupling assays on CHMO and six mutants that target four residues that mediate interactions between the Control Loop and the remainder of the enzyme. These data suggest a link between the Loop and the enzyme's structure and catalytic properties. This link may be useful in directing future enzyme engineering efforts. Furthermore, some experiments were repeated on another BVMO, cyclopentadecanone monooxygenase (CPDMO), that acts on larger substrates [21], demonstrating that these properties may be generally applicable to the BVMOs.

\section{MATERIALS AND METHODS}

\section{$2.1 \quad$ Small-Angle X-ray Scattering}

\subsubsection{Sample Preparation and Data Collection}

Samples of CHMO, its mutants, and CPDMO were prepared for SAXS data collection by adding appropriate amounts of ligands to the protein sample ( $15 \mathrm{mg} / \mathrm{mL}$ protein stock solutions), and then diluting the sample to $10 \mathrm{mg} / \mathrm{mL}$. These samples were microdialysed overnight against buffer with identical ligands, but without any protein. For CPDMO samples, $2 \mathrm{mM}$ Tris(2carboxy)phosphine (TCEP) was also added to both the samples and the buffer. Ligand 
concentrations for each sample are given in Table S5. Of the CHMO mutants, only W492A was analyzed with substrate and product.

Following dialysis, the samples were quantified using UV absorbance. Using the dialysis buffer, each sample was diluted 1:2 and 1:4. Data were collected at the SIBYLS beamline (beamline 12.3.1) at the Advanced Light Source using 0.5, 1.0, 2.0, and 4.0 second exposures. Data were collected from dialysis buffer with the same exposure times, and these buffer scattering curves were used for buffer subtraction.

In addition, a titration of CHMO with $\mathrm{NADP}^{+}$was prepared using a series of CHMO samples with increasing $\mathrm{NADP}^{+}$concentrations. Microdialysis was performed as previously described, but by diluting a $\sim 3 \mathrm{mg} / \mathrm{mL}$ stock solution to $2 \mathrm{mg} / \mathrm{mL}$, with $\mathrm{NADP}^{+}$concentrations ranging from $0 \mathrm{mM}$ to $10 \mathrm{mM}$. Following dialysis, the protein samples were quantified, and the $\mathrm{OD}_{260 \mathrm{~nm}}$ of the buffer was used to quantify the exact concentration of $\mathrm{NADP}^{+}$in the samples. Data were collected on a SAXSess $\mathrm{mc}^{2}$ nanostructure analysis system equipped with an Anton Paar $\mathrm{GmbH}$ detector for both the dialysed sample and the dialysis buffer. All data collection was performed at $4^{\circ} \mathrm{C}$. For each sample and buffer, a series of 36010 second exposures were measured and then averaged. In addition, dark current images of the same length were measured.

\subsubsection{BVMO Ligand Comparison Primary Data Analysis}

The data from each of the sets of scattering curves were scaled to the protein concentration. Curves in which the detector was saturated or the data exhibited pronounced concentration dependent effects were omitted from the analysis. The remaining curves were merged in PRIMUS [24] such that noisy data at higher q-ranges and interparticle repulsion at low q-ranges were eliminated from the final, merged scattering curves. The curves were then normalized to $\mathrm{I}(0)$. The radius of gyration $\left(\mathrm{R}_{\mathrm{g}}\right)$ was determined using the Guinier plot in PRIMUS [24]. The particle distance distribution function was then evaluated for each merged, normalized scattering curve using GNOM [25], and the maximum interatomic distance $\left(\mathrm{D}_{\max }\right)$ was determined. The Porod volume and Porod exponent were determined using ScÅtter (Rodic \& Rambo, Www.bioisis.net). SAXS data collection and analysis statistics are presented in Table 1 (CHMO), Table S1 (CHMO mutants), and Table S2 (CPDMO). The scattering data were deposited at the Small Angle Scattering Biological Database (SASBDB). 
Table 1: SAXS data collection and analysis statistics for CHMO

\begin{tabular}{|l|l|l|l|l|}
\hline & CHMO & $\begin{array}{l}\text { CHMO }+ \\
\mathrm{NADP}^{+}\end{array}$ & $\begin{array}{l}\text { CHMO }+\mathrm{NADP}^{+}+ \\
\text {cyclohexanone }\end{array}$ & $\begin{array}{l}\text { CHMO + NADP } \\
\varepsilon \text {-caprolactone }\end{array}$ \\
\hline Guinier $\mathrm{R}_{\mathrm{g}}(\AA)$ & $26.94 \pm 0.06$ & $25.95 \pm 0.14$ & $25.39 \pm 0.05$ & $25.33 \pm 0.04$ \\
\hline Real space $\mathrm{R}_{\mathrm{g}}(\AA)^{*}$ & $27.43 \pm 0.03$ & $26.08 \pm 0.02$ & $25.70 \pm 0.02$ & $25.59 \pm 0.01$ \\
\hline $\mathrm{D}_{\max }(\AA)^{*}$ & 93 & 80 & 78 & 75 \\
\hline Porod volume $\left(\AA^{3}\right)^{\dagger}$ & $1.1 \times 10^{5}$ & $9.9 \times 10^{4}$ & $1.0 \times 10^{5}$ & $9.9 \times 10^{4}$ \\
\hline Porod exponent ${ }^{\dagger}$ & 3.7 & 3.9 & 3.8 & 3.8 \\
\hline Total & 0.696 & 0.735 & 0.702 & 0.672 \\
\hline EOM $\chi^{\ddagger}$ & 4.3 & 1.3 & N/D & N/D \\
\hline SASBDB ID & SASDBA5 & SASDBB5 & SASDBC5 & SASDBD5 \\
\hline
\end{tabular}

Calculated by GNOM (Svergun, 1992).

${ }^{\dagger}$ Calculated by ScÅtter (Rodic \& Rambo, www.bioisis.net).

Calculated by EOM using the $\mathrm{CHMO}_{\text {Closed }}$ model in random mode without any ligands (Bernadó et al., 2007).

\subsubsection{NADP ${ }^{+}$Titration Data Analysis}

Data were analysed using SAXSquant (Anton Paar). The dark current was subtracted from all sample and buffer scattering curves, which were then normalized to the intensity of the attenuated direct beam. The appropriate buffer curve was then subtracted from the sample scattering curves. A Porod plot was constructed in order to evaluate any constant background scattering, and this constant was subtracted from the scattering curve as appropriate. The data were then truncated to a q-range of $0.17 \mathrm{~nm}^{-1}$ to $2.00 \mathrm{~nm}^{-1}$ and desmeared. The $\mathrm{R}_{\mathrm{g}}$ was determined using the Guinier plot in PRIMUS [24].

\subsection{Isothermal Titration Calorimetry}

ITC experiments were performed using a MicroCal iTC200 (GE Healthcare) at $25^{\circ} \mathrm{C}$. CHMO was loaded into the ITC cell, and $\mathrm{NADP}^{+}$was loaded into the syringe. Twenty $2 \mu \mathrm{L}, 4$ second injections were performed, with 150 seconds between injection to allow the system to return to the baseline. For each syringe concentration that was used, the same concentration of NADP ${ }^{+}$ 
was injected into $50 \mathrm{mM}$ Tris $\mathrm{pH} 8.0$ buffer as a background control under identical experimental conditions.

The ITC peaks were integrated using Origin 7.0. The background control data were subtracted from the experimental data. The data were fitted to the ITC One Site model using non-linear regression in Origin 7.0. Based on the values obtained for number of binding sites per protein $(\mathrm{N})$, the concentration was re-calculated to account for inaccuracies in protein concentration quantification by its $\mathrm{OD}_{280 \mathrm{~nm}}$. Due to the relatively low affinities for CHMO and its mutants, it was found that there was a strong dependence between the protein concentration, the fitted value of $\mathrm{N}$, and the $\Delta \mathrm{H}$, while the $\mathrm{K}_{\mathrm{d}}$ showed much better stability compared to these parameters [26]. In order to avoid over-parameterization of the data, $\mathrm{N}$ was fixed to 1 , as $\mathrm{CHMO}$ is known to bind to $\mathrm{NADP}^{+}$with 1:1 stoichiometry.

At least two independent ITC experiments were performed for each mutant. The ITC parameters were averaged to obtain the final values reported in Table S3.

\subsection{Uncoupling Assay}

The non-productive uncoupling of NADPH consumption from conversion of a substrate to a product can be measured by quantifying the amount of product produced in the presence of limiting NADPH and an excess of cyclohexanone. For each mutant, in triplicate, $3.0 \mathrm{mM}$ cyclohexanone was mixed with $1.5 \mathrm{mM}$ NADPH in $3.0 \mathrm{~mL}$ of $50 \mathrm{mM}$ Tris $\mathrm{pH}$ 8.0. The reaction was started by adding enzyme to each reaction to a final concentration of $100 \mu \mathrm{g} / \mathrm{mL}$ for the wild-type and $300 \mu \mathrm{g} / \mathrm{mL}$ for the mutants. The reactions were incubated at $4^{\circ} \mathrm{C}$ for several days, and the consumption of NADPH was monitored based on the $\mathrm{OD}_{340 \mathrm{~nm}}$. Controls without substrates were used to monitor the consumption of NADPH in the absence of cyclohexanone. Extraction controls of cyclohexanone and $\varepsilon$-caprolactone without enzyme or NADPH were used to assess the efficiency of extraction of the two compounds.

Once the NADPH was depleted based on $\mathrm{OD}_{340 \mathrm{~nm}}$, an equal volume of ethyl acetate was mixed with each reaction to extract the cyclohexanone and $\varepsilon$-caprolactone. The organic layer was dried over sodium sulfate and filtered. The samples were analyzed using gas chromatography on an Agilent 5975C Series inert XL EI/CI MSD with a Triple-Axis Detector equipped with a Varian Chirasil-Dex CB column ( $25 \mathrm{~m}$ x $0.25 \mathrm{~mm}$ x $0.25 \mu \mathrm{m})$. A $1 \mu \mathrm{L}$ volume from the extracted 
sample was injected under pulse split (20:1) condition with $40 \mathrm{~mL} / \mathrm{min}$ helium flow. The following gas chromatography conditions were used: $70^{\circ} \mathrm{C}$ for 2 minutes, $8^{\circ} \mathrm{C} /$ minute until $150^{\circ} \mathrm{C}$, and $150^{\circ} \mathrm{C}$ for 1 minute. The identity of the resulting peaks was confirmed using the MS data, and the cyclohexanone and $\varepsilon$-caprolactone peak areas were integrated.

The average adjusted uncoupling ratio is a measure of the degree of conversion of cyclohexanone to $\varepsilon$-caprolactone, accounting for the difference in extraction efficiency between cyclohexanone and $\varepsilon$-caprolactone. The ratio was calculated as follows:

$$
\begin{aligned}
& \text { Adjusted Uncoupling Ratio } \\
& \qquad=\frac{\text { Integrated Area of Cyclohexanone Peak }}{\text { Integrated Area of E-Caprolactone Peak }} \\
& \quad \times \frac{1}{\text { Extraction Efficiency Ratio }}
\end{aligned}
$$

where the extraction efficiency ratio is the ratio of integrated areas obtained from an equimolar solution of cyclohexanone and $\varepsilon$-caprolactone.

At the beginning of the reactions, twice as much cyclohexanone was present as NADPH. As such, if the reaction occurred with $100 \%$ efficiency, half of the cyclohexanone would be converted to $\varepsilon$-caprolactone, resulting in an Adjusted Uncoupling Ratio of 1.0. Controls performed in the absence of enzyme showed almost no background NADPH consumption, indicating that all of the NADPH was consumed by the active enzyme.

\section{RESULTS}

\subsection{Small Angle X-ray Scattering}

\subsubsection{NADP ${ }^{+}$Decreases the Radius of Gyration $\left(R_{g}\right)$ of BVMOs}

When the raw scattering curves of CHMO and CPDMO with their various ligands present were compared, they were found to be fairly similar, indicating that any conformational changes that occurred upon addition of $\mathrm{NADP}^{+}$, substrate, or product are relatively small (Fig. 2ac). Based on the particle distance distribution functions calculated from those curves (Fig. 2bd), however, it was possible to detect subtle changes in the overall particle size based on the $R_{g}$ and the $D_{\max }$. For both $\mathrm{CHMO}$ and CPDMO, addition of $\mathrm{NADP}^{+}$triggered a significant drop in both $\mathrm{R}_{\mathrm{g}}$ and 


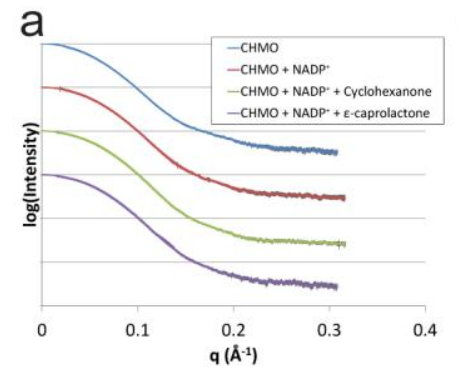

b

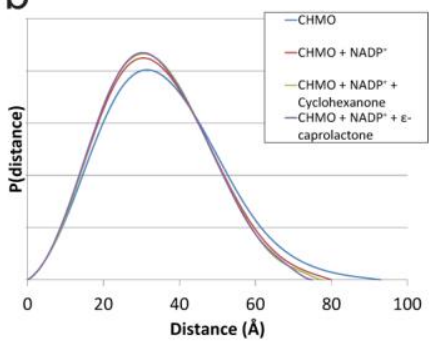

C

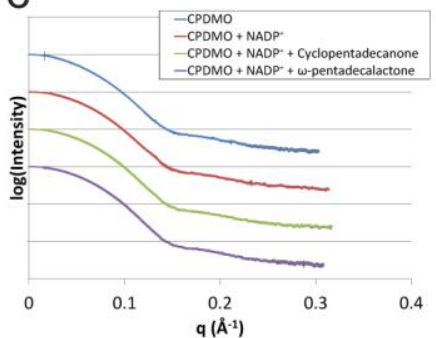

d

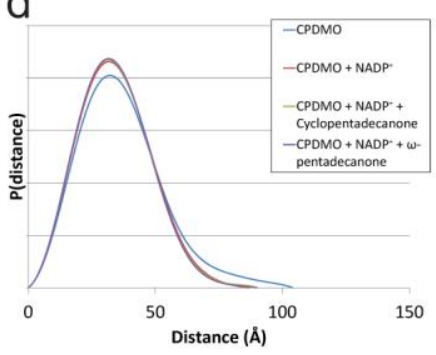

Fig. 2. Raw SAXS curves and probability distance distribution plots for (a, b) CHMO and (c, d) CPDMO with and without ligands. The intensities are plotted on a relative log scale. Error bars are shown as vertical, dark grey lines.
$D_{\text {max }}$. Further addition of either the ketone substrate or lactone product did result in a small but significant further decrease in $\mathrm{R}_{\mathrm{g}}$, though whether such a small change is mechanistically important is not clear. Of note, the smallest $R_{\mathrm{g}}$ for CHMO is still significantly higher than that calculated from the $\mathrm{CHMO}_{\text {Closed }}$ structure using CRYSOL [22].

In order to further probe the role of the Control Loop in these structural changes, a series of mutants (K328A, W492A, K328A-W492A, N497A, K501A, and N497A-K501A) that disrupt key polar contacts between the Loop and the rest of the protein were made. It should be noted that the enzyme is expected to be saturated with $\mathrm{NADP}^{+}$for all of the mutants, eliminating a decrease in affinity as a possible cause of any observed changes. Intriguingly, all four single mutants and both double mutants all exhibited the same trend when $\mathrm{NADP}^{+}$was added to the samples (Fig. 3, Fig. S1). In all cases, there was a substantial reduction in the $\mathrm{R}_{\mathrm{g}}$ and $\mathrm{D}_{\max }$ when $\mathrm{NADP}^{+}$was

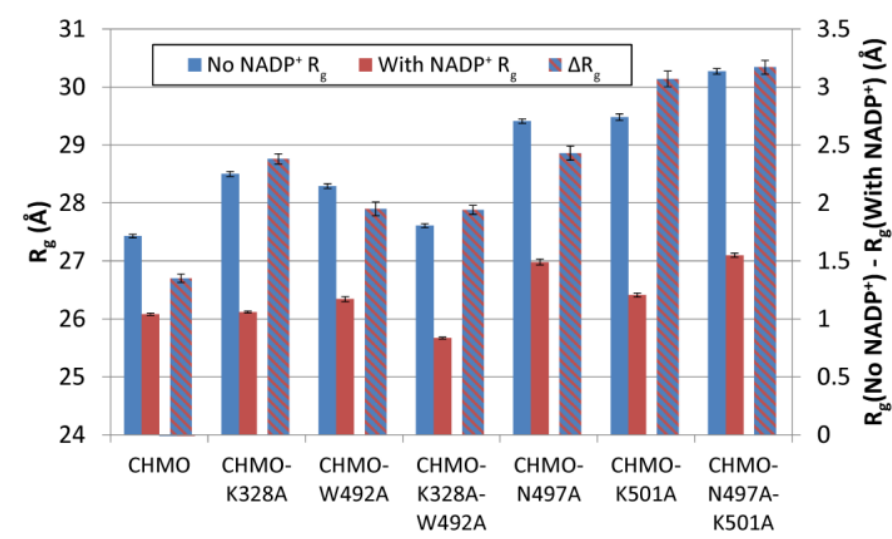

Fig. 3. Radius of gyration analysis for $\mathrm{CHMO}$ and its mutants. The $\mathrm{R}_{\mathrm{g}}$ is indicated in the absence and presence of NADP ${ }^{+}$. In addition, the difference between the $\mathrm{R}_{\mathrm{g}}$ in the absence and presence of $\mathrm{NADP}^{+}$is plotted using the right-hand axis. added, indicating that binding of $\mathrm{NADP}^{+}$ continued to trigger structural changes in the enzyme with all of the mutants. When comparing the magnitudes of these changes, as well as the $\mathrm{R}_{\mathrm{g}}$ values in the presence and absence of $\mathrm{NADP}^{+}$, however, it can be seen that the mutations appear to have a more pronounced effect on the enzyme's structure in the absence of $\mathrm{NADP}^{+}$. This is also shown when looking at the $\Delta R_{g}$ values (difference 
between the $\mathrm{R}_{\mathrm{g}}$ in the absence and presence of $\mathrm{NADP}^{+}$), which shows the smallest $\Delta \mathrm{R}_{\mathrm{g}}$ for the wild-type. In general, the wild-type enzyme had the smallest value for $\mathrm{R}_{\mathrm{g}}$, both in the presence and in the absence of $\mathrm{NADP}^{+}$. In the absence of $\mathrm{NADP}^{+}$, a substantial increase in the $\mathrm{R}_{\mathrm{g}}$ of the mutants of $0.8-3.0 \AA$ was observed. In contrast, in the presence of $\mathrm{NADP}^{+}$, the changes in $\mathrm{R}_{\mathrm{g}}$ were less than $0.4 \AA$, representing a very modest change, with the K328A-W492A mutant actually having a lower $\mathrm{R}_{\mathrm{g}}$ by $0.4 \AA$. There were three notable exceptions to this. In the absence of NADP ${ }^{+}$, the K328A-W492A mutant showed only a modest increase in $\mathrm{R}_{\mathrm{g}}$ of less than $0.2 \AA$. In the presence of $\mathrm{NADP}^{+}$, the $\mathrm{R}_{\mathrm{g}}$ of both N497A and N497A-K501A showed a substantial increase of about $1.0 \AA$, putting them at a similar level as the wild-type in the absence of

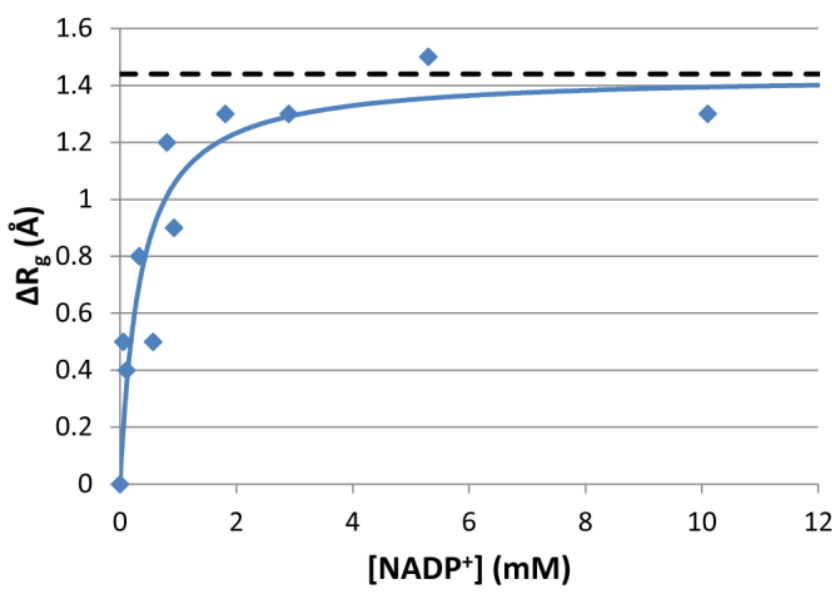

Fig. 4. Change in $\mathrm{R}_{\mathrm{g}}$ of CHMO with the titration of $\mathrm{NADP}^{+}$. The data are fitted to the general ligand binding equation. An asymptote corresponding to the maximum $\Delta \mathrm{R}_{\mathrm{g}}$ is indicated by the dashed line.
$\mathrm{NADP}^{+}$.

To show that this change is related to the degree of binding of $\mathrm{NADP}^{+}$, a series of SAXS curves were measured while titrating in $\mathrm{NADP}^{+}$(Fig. 4). A gradual decrease in the $R_{g}$ allowed the generation of a binding saturation curve. While the data clearly demonstrate a classical binding curve, the small difference between the measured $R_{g}$ values relative to the error in the $\mathrm{R}_{\mathrm{g}}$ measurements precludes obtaining statistically meaningful binding parameters from these data. The data are consistent with a $\mu \mathrm{M}$-range binding affinity, as derived from ITC experiments described below.

\subsubsection{CHMO Does Not Unfold in the Absence of NADP ${ }^{+}$}

The observed differences between the samples upon addition of $\mathrm{NADP}^{+}$, and in particular the reduction in $\mathrm{R}_{\mathrm{g}}$ and $\mathrm{D}_{\max }$, imply that the protein either becomes more compact or exhibits a lower degree of global protein flexibility. A variety of SAXS flexibility analyses were performed to assess whether protein unfolding or discrete sampling of multiple conformations was occurring 
in the $\mathrm{NADP}^{+}$-free state. This is described in more detail in the Supporting Information. In summary, the changes observed in the absence of ligands and upon the introduction of mutations are at least partly due to increased flexibility of CHMO. There are, however, no indications of major protein unfolding, suggesting that this flexibility is due to increased sampling of a number of fairly discrete conformational states, rather than changes in the degree of global protein foldedness.

\subsubsection{Structural Basis of the Changes in the SAXS Curves}

A variety of conformational changes could account for the increased compactness observed upon addition of $\mathrm{NADP}^{+}$. In order to assess the role of the Control Loop in these conformational changes, we used ensemble modeling of the loop with EOM to attempt to model these changes. A detailed description of the EOM results can be found in the Supporting Information. In summary, when fitted to the $\mathrm{NADP}^{+}$-bound curve, a reasonably good fit to the data was obtained with a major $\mathrm{R}_{\mathrm{g}}$ peak at around $25.5 \AA$, and sometimes a minor $\mathrm{R}_{\mathrm{g}}$ peak closer to $26.0 \AA$. Conversely, the opposite trend was observed when the data were fitted to the $\mathrm{NADP}^{+}$-free curve, with the major peak at $26.0 \AA$ and the minor peak sometimes obtained at $25.5 \AA$. It should be noted that the quality of the fit was significantly worse in the $\mathrm{NADP}^{+}$-free case, likely indicating that the crystal structures of CHMO do not correctly model the enzyme's global conformation in the absence of NADP ${ }^{+}$. Indeed, normal mode analysis with SREFLEX [23] (not shown) suggests that rotation of the NADPH-binding and/or helical domains, in additional to changes in the Control Loop, may be necessary to fully account for the $\mathrm{NADP}^{+}$-free scattering curve of wild-type CHMO.

It should be noted that, in contrast to the wild-type $\mathrm{NADP}^{+}$-free sample, none of the modeling techniques that we attempted were able to satisfactorily account for the very large increase in $\mathrm{R}_{\mathrm{g}}$ observed in some of the mutants. This suggests that domain rotations beyond what has been observed crystallographically may be occurring.

\subsection{Isothermal Titration Calorimetry}

The final ITC parameters are reported in Table S3. The affinity of the CHMO mutants with respect to $\mathrm{NADP}^{+}$was lower in all cases than the wild-type enzyme (Fig. 5). The K328A mutation had by far the greatest effect, with an increase in $\mathrm{K}_{\mathrm{d}}$ of almost a full order of 
magnitude. The K328A-W492A had a similar, slightly larger $\mathrm{K}_{\mathrm{d}}$ than the K328A single mutant. Of the remaining mutants, the N497A mutation had only a very minor effect on NADP ${ }^{+}$affinity, whereas W492A, K501A, and N497A-K501A showed an increase of roughly a factor of 2.

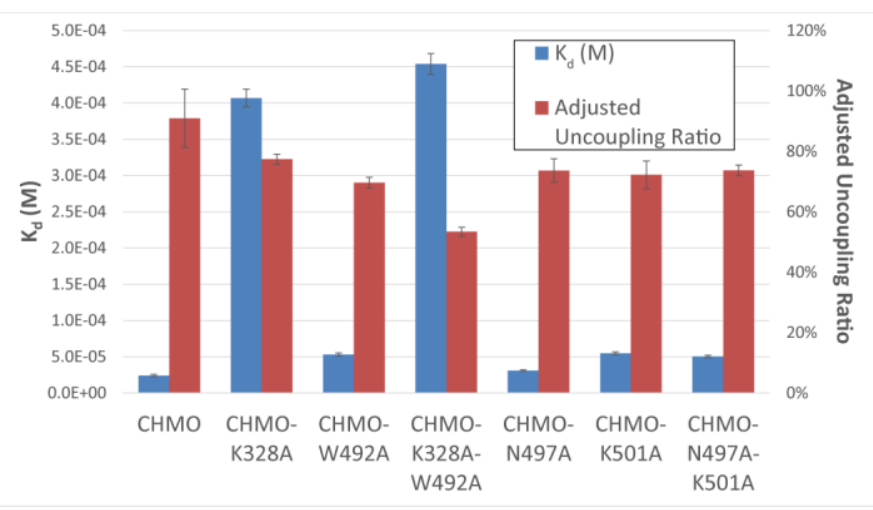

Fig. 5. $K_{d}$ and adjusted uncoupling ratio. The $K_{d}$ (left axis) and adjusted uncoupling ratio (right axis) derived from the ITC and uncoupling experiments, respectively, are shown for CHMO and its mutant.
Unlike the $\mathrm{K}_{\mathrm{d}}$ data, the enthalpy data proved to be somewhat unreliable, due to its high dependence on relatively small changes in the value of N. It is notable, however, that the two mutants with largest change in $\mathrm{K}_{\mathrm{d}}$, K328A and K328AW492A, both were less exothermic than the wild-type, whereas the other four mutants were more exothermic, with $\mathrm{NADP}^{+}$binding to the W492A mutant being the most exothermic interaction of

the wild-type and all the mutants.

\subsection{Uncoupling Assay}

Under conditions of excess substrate at saturating concentrations, the enzyme's ability to consume NADPH in a productive vs. non-productive manner can be monitored based on the amount of substrate converted to product. All of the NADPH should be consumed in the presence of excess substrate. In productive reaction cycles, substrate will bind to the peroxyanion intermediate form of the enzyme and be converted to the product. In nonproductive reaction cycles, no lactone will be formed. As such, the degree of conversion of substrate to product reflects the ratio of productive to non-productive reaction cycles. It must be emphasized that over the timescale of this experiment, controls with NADPH in the absence of any enzyme did not demonstrate any appreciable consumption of NADPH, indicating that all of the NADPH was consumed by the active enzyme. As this experiment examined the amount of production of $\varepsilon$-caprolactone compared to the consumption of a constant amount of NADPH at the endpoint of the reaction, factors such as differing turnover rates, specific activity, quantity of active enzyme, and enzyme stability cannot be playing a role. 
Unsurprisingly, the wild-type enzyme has by far the highest conversion of substrate to product, with greater than $90 \%$ of NADPH being consumed in productive reaction cycles (Fig. 5). The two single mutants and their double mutant in the signature sub-loop (N497A, K501A, and N497A-K501A) were substantially less efficient than the wild-type, but did not seem to have an additive effect, with all three showing $70-75 \%$ of the NADPH being consumed in productive reaction cycles, and the difference between mutants not being statistically significant. In the active site sub-loop, K328A and W492A also showed significantly reduced efficiencies, with $77 \%$ and $70 \%$ of reaction cycles being productive. In contrast, to the non-additive effect in the signature sub-loop, the double mutant in the active site sub-loop showed much less efficiency than either single mutant, with only $53 \%$ of reaction cycles being productive.

These results are consistent with controls (not shown) in which the enzyme was incubated with NADPH, but no substrate. All of the NADPH was consumed within 24 hours in the presence of any of the mutants except K328A. In contrast, the NADPH in the presence of wild-type CHMO required nearly a week to be consumed under similar conditions. This again demonstrates that the mutants undergo non-productive reaction cycles much more frequently than the wild-type enzyme.

\section{DISCUSSION}

Since the report of the first BVMO crystal structures $[12,15]$, the loop that roughly spans residues 487 to 504, referred to here as the Control Loop, has been seen as potentially important in catalysis. This was corroborated by the identical Loop conformation observed in CHMO and PAMO $[15,17]$, as well as the near-identical conformation of the N-terminal half of the Control Loop observed in structures of OTEMO [15, 18], which we are calling the active site sub-loop. Observations of the Control Loop in both ordered and disordered states in spite of NADP ${ }^{+}$being bound in both structures strongly suggested that the Loop is present in a dynamic equilibrium between the crystallographically observed ordered state and one or more disordered states, with the binding of $\mathrm{NADP}^{+}$driving that equilibrium towards the ordered state. Indeed, crystal crosslinking studies suggested the possibility of this conformational variability in crystals prior to freezing [16]. In addition, mutating the CHMO Control Loop residue W492 impacted catalytic activity in terms of both activity [15] and stereospecificity [16]. The most obvious role for the Loop based on the available crystal structures is to assist in "locking" the NADP(H) cofactor in 
place. The change in stereospecificity observed in the W492A mutant, however, suggested a more complex role in catalysis [16]. Here, we aim to address this role using a structure-function approach.

It is possible to detect relatively subtle changes in the population- and time-averaged conformation of a protein using SAXS by monitoring the $\mathrm{R}_{\mathrm{g}}, \mathrm{D}_{\max }$, and a variety of flexibility statistics. To that end, we collected SAXS data for CHMO and CPDMO in the presence and absence of their cofactor, substrates, and products. Unsurprisingly, the addition of NADP ${ }^{+}$ resulted in a substantial reduction in the $\mathrm{R}_{\mathrm{g}}$ and $\mathrm{D}_{\max }$ of the protein, consistent with crystallographically-observed Control Loop ordering and domain rotations that are observed when comparing the $\mathrm{CHMO}_{\text {Closed }}$ and $\mathrm{CHMO}_{\text {Open }}$ crystal structures [15]. Protein flexibility statistics appear to preclude that this change could be caused by changes in protein "foldedness," and rather points to the enzyme sampling multiple discrete states in the absence of NADP ${ }^{+}$. This is consistent with multiple Control Loop conformations and NADPH-binding domain positions being sampled in the $\mathrm{NADP}^{+}$-free state. In other words, it appears that addition of $\mathrm{NADP}^{+}$shifts the equilibrium between the Closed and Open states of CHMO and CPDMO towards the more Closed conformations. It should be noted that these changes relate to the equilibrium average, without giving us direct information about conformational change kinetics. As both CHMO and CPDMO exhibited similar changes upon addition of $\mathrm{NADP}^{+}$, it is possible that our observations here can be generalized to other BVMOs.

While $\mathrm{NADP}^{+}$is bound to the BVMOs during the majority of their catalytic cycle, it remains possible that the NADPH-bound form, as well as the various states of the FAD cofactor, may alter the behavior of the Control Loop. Given that the substrate- and product-bound SAXS data are very similar to the $\mathrm{NADP}^{+}$-bound data, however, we feel that any differences in these states are likely too subtle to be differentiated in the SAXS experiments. Our broad conclusions are, therefore, likely valid across the range of NADP(H)-associated states.

To determine what role the Control Loop plays in these observed changes, we repeated the SAXS experiments with a number of CHMO mutants in which key Control Loop-protein interactions are disrupted. Surprisingly, only one of the four mutation sites that we targeted appeared to have a substantial effect on the SAXS curves in the presence of NADP ${ }^{+}$. This 
signature sub-loop mutant, N497A (as well as the N497A-K501A double mutant), had an $\mathrm{R}_{\mathrm{g}}$ in the presence of $\mathrm{NADP}^{+}$similar to the $\mathrm{R}_{\mathrm{g}}$ of the wild-type enzyme measured in the absence of $\mathrm{NADP}^{+}$. As part of the signature sub-loop, this residue does not form close contacts with the active site or $\mathrm{NADP}^{+}$, instead forming indirect interactions with the BVMO signature sequence. This suggests that in the presence of $\mathrm{NADP}^{+}$, the N497A mutation causes the Control Loop ordering/domain rotation equilibrium to shift to resemble that observed in the wild-type in the absence of NADP ${ }^{+}$.

Conversely, all of the mutations had a notable impact on the $\mathrm{R}_{\mathrm{g}}$ in the absence of NADP ${ }^{+}$. Consistent with N497's role in the presence of $\mathrm{NADP}^{+}$, the signature sub-loop mutations had a much more dramatic increase in $\mathrm{R}_{\mathrm{g}}$ than the active site sub-loop mutations. The magnitudes of these changes are likely to be too large, at least in the most extreme cases, to be caused exclusively by loop ordering and disordering. Given that the protein flexibility statistics do not suggest protein unfolding, we must therefore conclude that other conformational changes, such as the rotation of the NADPH-binding or helical domains, contribute to the larger $\mathrm{R}_{\mathrm{g}}$ in addition to the Control Loop's state. Given the larger influence of the signature sub-loop interactions in both $\mathrm{NADP}^{+}$states, it is reasonable to speculate that the signature sub-loop plays a structural role, likely by coordinating domain movements through its interactions with the BVMO signature sequence. The active site sub-loop, conversely, is playing a secondary role. That the conformational changes caused by the mutations in the active site sub-loop could propagate through the Control Loop to the signature sub-loop, and finally to the BVMO signature sequence, is one possible explanation for the impact of the active site sub-loop mutations on the $\mathrm{NADP}^{+}$-free state.

The fit between any of the SAXS data in the absence of $\mathrm{NADP}^{+}$and the available crystal structures of BVMOs, including the more open $\mathrm{CHMO}_{\mathrm{Open}}$ crystal structure and the $\mathrm{NADP}^{+}$-free PAMO structure, is relatively poor. This may indicate that CHMO is able to access more open conformations than the available crystal structures would suggest. Crystal packing may be limiting the conformational flexibility available to the enzyme, thereby preventing us from observing the most open conformations by crystallography so far. This may explain the ensemble modeling results (see Supporting Information) as well as our preliminary normal mode analysis results (not shown). We can speculate that the large $\mathrm{R}_{\mathrm{g}}$ values observed in the signature 
sub-loop mutant SAXS curves could be indicative of an increase in the population of these "wide open" conformations that are not crystallographically observed. The consistent disordering of the signature sub-loop in the OTEMO crystal structures may be an indication that the enzyme has evolved to more readily occupy these "wide open" conformations in order to accommodate its much larger, CoA-linked substrate [18].

Structural and functional studies of the Control Loop to date have suggested that it has a role in catalysis $[15,16]$. In spite of this, given that the structural changes triggered by disruption of Control Loop-protein contacts is far more pronounced in the non-catalytic $\mathrm{NADP}^{+}$-free state, it raises the question as to whether these changes are in fact relevant to the catalytically active protein states. To address this, we used two functional assays to determine whether these mutations have a measurable effect on catalysis. We used ITC experiments to detect changes in the binding affinity of $\mathrm{NADP}^{+}$to $\mathrm{CHMO}$ when Control Loop interactions were disrupted. The second experiment, the uncoupling assay, indicates what proportion of the FAD reduced by NADPH is used to productively turnover a cyclohexanone substrate to an $\varepsilon$-caprolactone product. It should be noted that this is independent of factors like reaction rates and protein stability, as NADPH is stable in the absence of enzyme under the reaction conditions, and in all cases, the NADPH was allowed to be consumed entirely by the enzyme. We can therefore be confident that the same number of FAD reduction events occurred in all of the uncoupling experiments.

The uncoupling assay clearly shows that, as expected, all six mutants are less efficient in using NADPH productively to perform the BVO reaction, as opposed to non-productively in the NADPH oxidase reaction. This demonstrates that these Control Loop interaction residues do play a role in catalysis. Notably, the mutations made in the active site sub-loop appear to have additive effects in the uncoupling experiments, whereas the mutations in the signature sub-loop do not. This suggests that while both sub-loops are implicated in the efficient consumption of $\mathrm{NADPH}$, the active site sub-loop has a more important role in that regard. It is possible that the structural disruption in one sub-loop is propagating through the Control Loop to influence the other. 
As previously stated, the most obvious role for the Control Loop to play based on the available crystal structures is to act as a "lid" on the active site when NADP(H) is bound. If the enzyme's inability to keep $\operatorname{NADP}(\mathrm{H})$ bound is the reason for the changes observed in the mutants, we would expect that the magnitude of these changes would be related to the decrease in NADP ${ }^{+}$ affinity, as measured by ITC. Strikingly, the K328A mutant has by far the greatest effect on $\mathrm{NADP}^{+}$affinity, but equal or smaller effects on the uncoupling assay and in the SAXS experiments than the other mutations. While we cannot exclude that the reduction in $\mathrm{NADP}^{+}$affinity is partly responsible for the changes we observed in the other experiments, the lack of correlation between $\mathrm{NADP}^{+}$affinity and the other results make it clear that it is not the most important factor. Having said that, the differences in the structural role of these residues in the absence and presence of $\mathrm{NADP}^{+}$indicate that the Control Loop plays a different role depending on $\mathrm{NADP}^{+}$binding.

The data presented here show that the Control Loop interactions disrupted in this study have an impact on structure, especially in the absence of $\mathrm{NADP}^{+}$, as well as on function, which has been highlighted by previous experiments targeting W492 [15, 16]. It appears that changes directly related to the core catalytic functions, namely co-factor binding, productive NADPH consumption, and substrate selection are mediated more strongly by the active site sub-loop. In contrast, the structural effects observed are mediated primarily by the signature sub-loop. This is not unexpected, given the proximity of the active site sub-loop to the active site and co-factor binding sites, as well as the signature sub-loop's proximity to an interdomain linker that in turn contacts the BVMO signature sequence. It does not explain, however, the smaller but detectable role of the signature sub-loop on "active site" effects as well as the active site sub-loop's impact on structure.

Given that the two sub-loops are covalently linked to each other, it is likely that the disruption of interactions in one sub-loop can lead to structural changes in the other, thereby triggering the minor effects that the more distant sub-loop has in each experiment. The high degree of sequence conservation in the BVMO signature sequence suggests that this sequence has more importance than simply contributing to the Rossmann fold of the NADPH-binding domain, although its distance from the active site appeared to preclude any catalytic role $[12,15]$. We 
speculate that the Control Loop structurally and functionally links the signature sequence and the active site through its interactions with both regions of the protein.

Our previous study demonstrated the Control Loop has potential to be an interesting protein design target, owing to the improved stereospecificity observed in the W492A mutant, albeit at the cost of enzyme efficiency [16]. Here, we demonstrate that active site sub-loop has a greater impact on active site effects, whereas the signature sub-loop has a greater impact on structural effects, but that both sub-loops are able to make contributions to both effects. With further study, it may be possible to modulate stereospecificity without negatively impacting enzyme efficiency through compensatory mutations in the two sub-loop regions. This may be a promising, underexplored avenue for future BVMO engineering efforts.

\section{CONCLUSIONS}

The complex domain and loop movements of the BVMOs have been implicated through kinetic studies as well as crystallographic studies. There has as of yet been no attempt to structurally characterize these changes in solution, however. We have shown that the large "Control Loop" between residues 487 and 504, which we have previously demonstrated to be important for catalysis [15] and have proposed to play a role in the domain and active site organization of the enzyme $[16,19]$, can be studied in solution by SAXS. We have shown that the addition of $\mathrm{NADP}^{+}$to $\mathrm{CHMO}$ and CPDMO cause the enzymes to adopt a more compact overall structure, likely due to a combination of the closing of the NADPH-binding domain and the ordering of the Control Loop. As both structural states are expected to be occupied with and without the ligands, this represents a shift in the equilibrium upon addition of NADP ${ }^{+}$. Furthermore, by disrupting several key Control Loop interactions using mutagenesis, we can demonstrate that the Loop affects $\mathrm{NADP}^{+}$affinity, the global protein structure, and the active site environment. We have proposed the division of the Loop into two sub-loops. The active site sub-loop has a greater effect on the active site environment, while the signature sub-loop has a greater effect on overall protein structure, likely through its indirect interaction with the BVMO signature sequence. Structural interactions between the two sub-loops, however, allow both parts of the Loop to play a role in both of these processes, thereby linking changes in the active site environment to changes in the global protein structure. These studies clearly demonstrate a more complex role for the Control Loop than previously anticipated. Given the similarities observed between 
CHMO and CPDMO, these observations may be extrapolated to many other BVMOs. A general understanding of BVMO flexibility will hopefully be useful in moving forward BVMO engineering efforts.

\section{ACKNOWLEDGMENTS}

We would like to thank the past and present members of the Berghuis and Lau labs for their help and suggestions. In addition, we would like to thank Dr. Anthony Mittermaier (McGill University) for his helpful comments for this manuscript, and Dr. Robert Rambo (Diamond Light Source) for his advice regarding the SAXS flexibility analyses. We would also like to thank Kevin Dyer and Kathryn Burnett, at the Advanced Light Source Structurally Integrated Biology for Life Sciences (SIBYLS) beamline (12.3.1), for collecting and performing the preliminary analysis on the synchrotron SAXS data. The GC/MS data were collected at the McGill/Goodman Cancer Research Centre Metabolomics Core Facility. We would like to thank Luc Choinière and Daina Avizonis for their help in developing and running the GC/MS protocol. This research was made possible by grants from the Natural Sciences and Engineering Research Council [NSERC RGPIN 183867-13] and the Canadian Institutes of Health Research [CIHR MOP-114889] awarded to A.M.B. B.J.Y. has held scholarships from the Natural Sciences and Engineering Research Council of Canada, McGill University, the CIHR Strategic Initiative in Chemical Biology, and the NSERC CREATE Program in Bionanomachines. A.M.B. holds a Canada Research Chair in Structural Biology. Support by Tianjin "Thousand Talents" Program for Senior International Scientists to P.C.K.L. is gratefully acknowledged. P.C.K.L. also would like to thank the Fonds Québécois de la recherche sur la nature et les technologies Center for Green Chemistry and Catalysis for partial support. High throughput SAXS data collection at SIBYLS is funded by the Integrated Diffraction Analysis (IDAT) program, supported by the DOE Office of Biological and Environmental Research, and from the National Institute of Health project MINOS RO1.

\section{APPENDIX}

Supplementary data, methods, and results to this article can be found online. 


\section{REFERENCES}

[1] A. Baeyer, V. Villiger, Einwirkung des Caro'schen Reagens auf Ketone, Ber. Dtsch. Chem. Ges., 32 (1899) 3625-3633.

[2] A. Baeyer, V. Villiger, Ueber die Einwirkung des Caro'schen Reagens auf Ketone, Ber.

Dtsch. Chem. Ges., 33 (1900) 858-864.

[3] N.A. Donoghue, D.B. Norris, P.W. Trudgill, The Purification and Properties of

Cyclohexanone Oxygenase from Nocardia globerula CL1 and Acinetobacter NCIB 9871, Eur. J. Biochem., 63 (1976) 175-192.

[4] N.A. Donoghue, P.W. Trudgill, The Metabolism of Cyclohexanol by Acinetobacter NCIB 9871, Eur. J. Biochem., 60 (1975) 1-7.

[5] V. Alphand, R. Wohlgemuth, Applications of Baeyer-Villiger Monooxygenases in Organic Synthesis, Curr. Org. Chem., 14 (2010) 1928-1965.

[6] H. Leisch, K. Morley, P.C.K. Lau, Baeyer-Villiger Monooxygenases: More Than Just Green Chemistry, Chem. Rev., 111 (2011) 4165-4222.

[7] Z.-G. Zhang, L.P. Parra, M.T. Reetz, Protein Engineering of Stereoselective Baeyer-Villiger Monooxygenases, Chem. Eur. J., 18 (2012) 10160-10172.

[8] C.C. Ryerson, D.P. Ballou, C.T. Walsh, Mechanistic Studies on Cyclohexanone Oxygenase, Biochemistry, 21 (1982) 2644-2655.

[9] D. Sheng, D.P. Ballou, V. Massey, Mechanistic Studies of Cyclohexanone Monooxygenase: Chemical Properties of Intermediates Involved in Catalysis, Biochemistry, 40 (2001) 1115611167.

[10] R. Criegee, Die Umlagerung der Dekalin-peroxydester als Folge von kationischem Sauerstoff, Liebigs Ann. Chem., 560 (1948) 127-135.

[11] D.E. Torres Pazmiño, B.-J. Baas, D.B. Janssen, M.W. Fraaije, Kinetic Mechanism of Phenylacetone Monooxygenase from Thermobifida fusca, Biochemistry, 47 (2008) 4082-4093. 
[12] E. Malito, A. Alfieri, M.W. Fraaije, A. Mattevi, Crystal Structure of a Baeyer-Villiger Monooxygenase, Proc. Natl. Acad. Sci. U. S. A., 101 (2004) 13157-13162.

[13] M.W. Fraaije, N.M. Kamerbeek, W.J.H. van Berkel, D.B. Janssen, Identification of a Baeyer-Villiger Monooxygenase Sequence Motif, FEBS Lett., 518 (2002) 43-47.

[14] M.L. Mascotti, W.J. Lapadula, M. Juri Ayub, The Origin and Evolution of Baeyer-Villiger Monooxygenases (BVMOs): An Ancestral Family of Flavin Monooxygenases, Plos One, 10 (2015) e0132689.

[15] I.A. Mirza, B.J. Yachnin, S. Wang, S. Grosse, H. Bergeron, A. Imura, H. Iwaki, Y. Hasegawa, P.C.K. Lau, A.M. Berghuis, Crystal Structures of Cyclohexanone Monooxygenase Reveal Complex Domain Movements and a Sliding Cofactor, J. Am. Chem. Soc., 131 (2009) 8848-8854.

[16] B.J. Yachnin, M.B. McEvoy, R.J.D. MacCuish, K.L. Morley, P.C.K. Lau, A.M. Berghuis, Lactone-Bound Structures of Cyclohexanone Monooxygenase Provide Insight into the Stereochemistry of Catalysis, ACS Chem. Biol., 9 (2014) 2843-2851.

[17] R. Orru, H.M. Dudek, C. Martinoli, D.E. Torres Pazmiño, A. Royant, M. Weik, M.W. Fraaije, A. Mattevi, Snapshots of Enzymatic Baeyer-Villiger Catalysis, J. Biol. Chem., 286 (2011) 29284-29291.

[18] H. Leisch, R. Shi, S. Grosse, K. Morley, H. Bergeron, M. Cygler, H. Iwaki, Y. Hasegawa, P.C.K. Lau, Cloning, Baeyer-Villiger Biooxidations, and Structures of the Camphor Pathway 2Oxo- $\Delta^{3}-4,5,5$-Trimethylcyclopentenylacetyl-Coenzyme A Monooxygenase of Pseudomonas putida ATCC 17453, Appl. Environ. Microbiol., 78 (2012) 2200-2212.

[19] B.J. Yachnin, T. Sprules, M.B. McEvoy, P.C.K. Lau, A.M. Berghuis, The Substrate-Bound Crystal Structure of a Baeyer-Villiger Monooxygenase Exhibits a Criegee-like Conformation, J. Am. Chem. Soc., 134 (2012) 7788-7795.

[20] K. Balke, S. Schmidt, M. Genz, U.T. Bornscheuer, Switching the Regioselectivity of a Cyclohexanone Monooxygenase toward (+)-trans-Dihydrocarvone by Rational Protein Design, ACS Chem. Biol., 11 (2016) 38-43. 
[21] H. Iwaki, S. Wang, S. Grosse, H. Bergeron, A. Nagahashi, J. Lertvorachon, J. Yang, Y. Konishi, Y. Hasegawa, P.C.K. Lau, Pseudomonad Cyclopentadecanone Monooxygenase Displaying an Uncommon Spectrum of Baeyer-Villiger Oxidations of Cyclic Ketones, Appl. Environ. Microbiol., 72 (2006) 2707-2720.

[22] D. Svergun, C. Barberato, M.H.J. Koch, CRYSOL - a Program to Evaluate X-ray Solution Scattering of Biological Macromolecules from Atomic Coordinates, J. Appl. Crystallogr., 28 (1995) 768-773.

[23] A. Panjkovich, D.I. Svergun, Deciphering conformational transitions of proteins by small angle X-ray scattering and normal mode analysis, Physical Chemistry Chemical Physics, In press (2016).

[24] P.V. Konarev, V.V. Volkov, A.V. Sokolova, M.H.J. Koch, D.I. Svergun, PRIMUS : a Windows PC-Based System for Small-Angle Scattering Data Analysis, J. Appl. Crystallogr., 36 (2003) 1277-1282.

[25] D.I. Svergun, Determination of the Regularization Parameter in Indirect-Transform Methods Using Perceptual Criteria, J. Appl. Crystallogr., 25 (1992) 495-503.

[26] W.B. Turnbull, A.H. Daranas, On the Value of c: Can Low Affinity Systems Be Studied by Isothermal Titration Calorimetry?, J. Am. Chem. Soc., 125 (2003) 14859-14866. 


\section{Graphical Abstract}

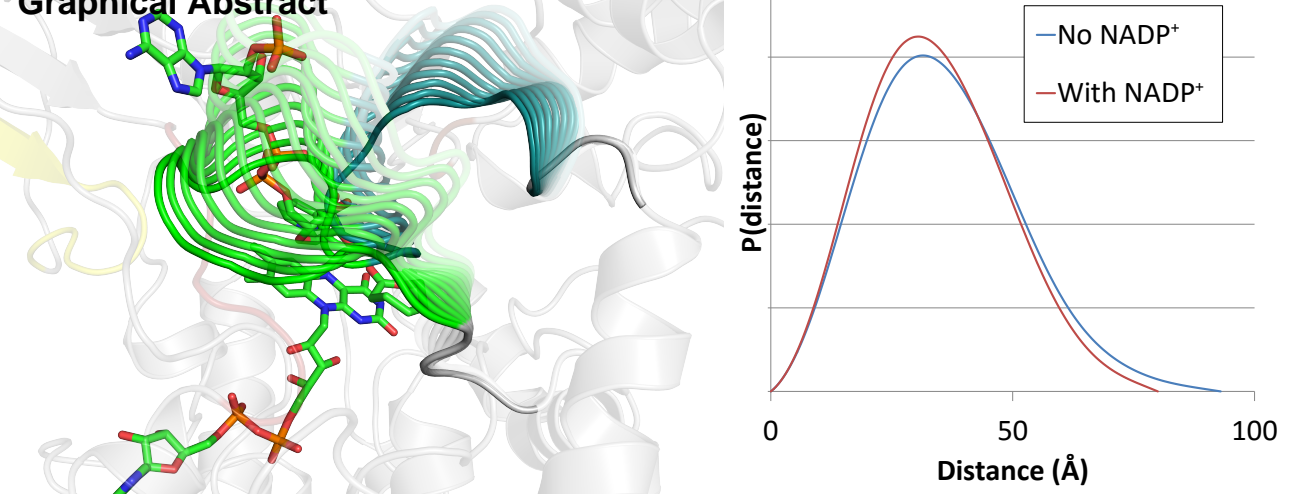

